\title{
Decreased Anterior Cingulate Myo-inositol/ Creatine Spectroscopy Resonance with Lithium Treatment in Children with Bipolar Disorder
}

\author{
Pablo Davanzo, M.D., M. Albert Thomas, Ph.D., Kenneth Yue, B.S., Thomas Oshiro, M.S.,
} Thomas Belin, Ph.D., Michael Strober, Ph.D., and James McCracken, M.D.

This project was designed to compare differences in brain proton spectra between children and adolescents with bipolar disorder (BPD) and gender and age-matched normal controls, and to measure changes in myo-inositol levels following lithium therapy, utilizing in vivo proton magnetic resonance spectroscopy $\left({ }^{1} H\right.$ MRS). A single voxel $(2 \times 2 \times 2 \mathrm{~cm} 3)$ was placed in brain anterior cingulate cortex for acquisition of the ${ }^{1} \mathrm{H}$ spectra at baseline and after acute ( 7 days) lithium administration in 11 children (mean age 11.4 years) diagnosed with $B P D$, and in 11 normal controls. Acute lithium treatment was associated with a significant reduction in the myo-inositol/creatine ratio. This decrement was also significant in lithium-responders when analyzed separate from non-responders. Compared to normal controls, BPD subjects showed a trend towards a higher myo-inositol/creatine during the manic phase. These preliminary data provide evidence that a significant reduction in anterior cingulate myo-inositol magnetic resonance may occur after lithium treatment, especially among responders. Follow-up studies involving a larger sample may allow us to confirm whether changes in myoinositol associated with acute lithium therapy persist in long-term clinical response of patients with and without lithium compliance.

[Neuropsychopharmacology 24:359-369, 2001] (C) 2001 American College of Neuropsychopharmacology. Published by Elsevier Science Inc.
KEY WORDS: Pre-adolescents; Mania; Hypomania; Lithium; Magnetic resonance spectroscopy

Bipolar affective disorder (BPD) is a severe, recurrent, potentially life-threatening illness (Tondo et al. 1998). Epidemiological data (Lewinsohn et al. 1995), suggest that the prevalence of juvenile-onset BPD may be as common as rates reported in adult populations, al-

From the Department of Psychiatry and Behavioral Sciences (PD, MS, JM), Department of Radiological Sciences (MAT, KY, TO), Department of Biostatistics (TB), School of Medicine, University of California, Los Angeles

Address correspondence to: Pablo Davanzo, M.D., Assistant Professor, Dept. of Psychiatry, UCLA School of Medicine, Room 48243C-UCLA Neuropsychiatric Institute, 760 Westwood Plaza, Los Angeles, CA 90024. Tel.:310-825-0469, Fax: 310-206-4446, E-mail: pdavanzo@mednet.ucla.edu

Received April 13, 2000; revised August 25, 2000; accepted September 14, 2000. though estimates vary widely (Costello et al. 1996). Given the increased frequency of lithium use among children and adolescents (Ryan et al. 1999) there is a clear need to assess the effects of lithium in pediatric populations. Children may have greater lithium resistance than adults (Himmelhoch and Garfinkel 1986; Hsu 1986), and it may take up to six weeks to determine, based on clinical signs and symptoms, whether a child is responding to treatment. Biological markers of treatment response would be extremely useful, particularly in these times of limited inpatient resources. The present study measured the magnetic resonance of myo-inositol and other metabolites with ${ }^{1} \mathrm{H}$ MRS in pediatric subjects diagnosed with BPD, before and during lithium therapy, with baseline comparison to normal controls individually matched for age and gender. We hypothesized that lithium would have an effect on the 
magnetic resonance of myo-inositol in the anterior cingulate cortex in children with BPD, as measured by in vivo proton magnetic resonance spectroscopy ( ${ }^{1} \mathrm{H}$ MRS). Secondarily, we postulated that a decrease in myo-inositol signal in response to lithium might be a predictor of treatment efficacy.

Lithium has traditionally been the preferred treatment of the manic phase of bipolar illness in adults, children (Geller and Luby 1997), and adolescents (Strober et al. 1995) with BPD. Open-label (Hsu 1986; Carlson et al. 1992; Strober et al. 1995), and one doubleblind placebo-controlled study of adolescents with substance abuse and mania (Geller et al. 1998) suggest that juveniles with BPD may have somewhat reduced benefit from acute lithium therapy compared with adults. Nevertheless, lithium continues to be widely prescribed among children and adolescents with BPD (Geller and Luby 1997) as well as for young patients presenting with excessive irritability (Fava 1997) and extreme affective dysregulation (Vitiello and Stoff 1997).

The precise neurobiological mechanisms whereby lithium reduces acute manic excitement and protects against recurrence of illness remain uncertain. It is apparent that lithium exerts an effect on intracellular second-messenger systems in which activated receptorligand complexes stimulate the turnover of inositolcontaining phospholipids (del Rio et al. 1998; Feldman et al. 1997; Murray and Greenberg 1997; Soares et al. 1999). Lithium's inhibitory action on receptor-mediated signal-transduction pathways has been demonstrated in vitro (Atack et al. 1995) showing that it reduces myoinositol levels by non-competitively inhibiting the enzyme inositol monophosphate, a catalyst for converting inositol monophosphate hydroxyls to myo-inositol (Manji et al. 1996). Since the phosphoinositide cycle (PI) regulates a wide variety of neuronal functions, including intracellular calcium mobilization and protein $\mathrm{ki}-$ nase C (PKC) activity (Feldman et al. 1997), lithiuminduced modification of the PI cycle has been proposed as a potential therapeutic mechanism underlying its mood-stabilizing effect (Berridge et al. 1982; del Rio et al. 1998; Murray and Greenberg 1997; Soares et al. 1999). Although brain tissue can synthesize myo-inositol de novo, the ability of neurons to maintain a steady-state supply of cytosolic myo-inositol appears to be crucial to the resynthesis of phosphoinositides, and, conceivably, to the membrane receptor response to stimulation and neuronal homeostasis. Dampening of PI-mediated signal transduction in excitatory neurons would therefore, result in antimanic effect (Feldman et al. 1997).

However, clinical findings bearing on PI signal transduction activity in BPD are inconsistent (Feldman et al. 1997). Most information about PI metabolism in BPD derives from studies using tissues other than CNS (Atack et al. 1995; Feldman et al. 1997). Indirect evidence for altered PI metabolism in individuals with
BPD has been suggested by a reduction in cytosolic and membrane-associated PKC activities in platelets of lithium-treated subjects with BPD (Friedman et al. 1993); and by elevated platelet membrane phosphatidylinositol-4,5-bisphosphate (PIP2) in medication-free patients with BPD during the manic phase (Brown et al. 1993).

Indirect in vivo measurement of brain inositol metabolism in adult patients treated with lithium has been attempted through measuring phosphomonoester peaks with phosphorus $\left({ }^{31} \mathrm{P}\right)$ magnetic resonance spectroscopy (MRS) (Kato et al. 1995), proton $\left({ }^{1} \mathrm{H}\right)$ MRS (Kato et al. 1996; Silverstone et al. 1996) and lithium MRS (Gonzalez et al. 1993; Kato et al. 1993; Sachs et al. 1995) (involving a lithium-sensitive coil). In vivo ${ }^{1} \mathrm{H}$ MRS is a non-invasive technique for measuring metabolite concentrations in living tissue (Renshaw et al. 1995). The major compounds observed in ${ }^{1} \mathrm{H}$ MRS spectra of the brain are myo-inositol (mI), choline moieties (Cho), creatine (Cr), and N-acetyl-aspartate (NAA) (Miller 1991). Sharma and colleagues (Sharma et al. 1992) reported an elevation of the inositol/Cr ratios in the basal ganglia of adult patients treated with lithium, a finding that appears to contradict in vitro findings showing a decrement of inositol with lithium treatment in animals (Manji et al. 1996). Likewise, Silverstone and colleagues (Silverstone et al. 1996) reported that chronic lithium did not alter human myo-inositol/Cr peak ratios as measured by ${ }^{1} \mathrm{H}$ MRS, in a study of seven volunteer subjects receiving lithium compared to four volunteers receiving placebo over seven days. These studies appear to contradict the hypothesis that lithium significantly affects brain concentrations of myo-inositol or phosphomonoesters. However, the possibility cannot be discounted that these earlier failures to detect changes in brain metabolism (ie. myo-inositol and phosphomonoester concentrations) were due to small samples, or studying patients who were on lithium at serum levels in the lower end of the therapeutic range (Sachs et al. 1995).

Recently, Moore and colleagues (Moore et al. 1999) used ${ }^{1} \mathrm{H}$ MRS to study lithium's effects on in vivo brain inositol and choline levels in adult depressed patients with BPD. Brain myo-inositol and choline levels were measured at three time points: at baseline, after 5-7 days of acute lithium treatment and 3-4 weeks of initiation of lithium. Significant decreases, averaging 30\%, were observed in levels of myo-inositol in the right frontal lobe following acute lithium administration, which persisted through one month of treatment. Reduction in myo-inositol resonance was observed prior to onset of observed clinical improvement, suggesting a marker for the beginning of a cascade of neuronal changes involving PKC regulation, and gene expression of the major PKC substrate, myristoylated alanine-rich C-kinase substrate (MARCKS), all potential sites for lithium action in the brain. 
At present, the effect of lithium on myo-inositol in children with BPD remains largely unknown. We are not aware of any studies examining this measurement so far. The present study sought to assess this effect with in vivo ${ }^{1} \mathrm{H}$ MRS in 11 pediatric subjects with BPD tested before and after one week of lithium therapy, including baseline comparison with 11 normal controls individually matched for age and gender.

\section{SUBJECTS AND METHODS}

The study was reviewed and approved by the UCLA Human Subjects Protection Committee. All patients, parents, and controls provided written informed consent and assent. Subjects received a semi-structured interview with the Mini International Neuropsychiatric Interview for Children and Adolescents (Mini-Kid) (Sheehan et al. 1998), and had Young Mania Rating Scale (YMRS) (Young et al. 1978) scores above 12 prior to enrollment. A child psychiatrist (PD), who reviewed the inpatient's team assessment and research measures to reach a best estimate diagnosis, ascertained diagnoses. All patients received a medical and psychiatric history, as well as a complete physical and neurological examination. Routine laboratories screen and thyroid screen were obtained on all patients. Patients were excluded if their age was below 5 or over 18, if they had history of prior lithium treatment failure, if they met diagnostic criteria for mental retardation, pervasive developmental disorder, or schizophrenia. They were also excluded if they had an uncontrolled medical disorder, including thyroid disease, seizure disorder, or neurological disease. One patient with well-controlled diabetes was allowed to participate in the study. Patients with implanted metallic devices or significant claustrophobia were excluded. The majority $(n=9)$ of the sample received a diagnosis of BPD mixed type. Two patients received a diagnosis of BPD Type II. Five of eleven patients $(45.4 \%)$ also received the diagnosis of Attention Deficit Hyperactivity Disorder (ADHD) and four $(36.3 \%)$ the diagnosis of Oppositional Defiant Disorder (ODD). One child satisfied DSM-IV diagnostic criteria for Chronic Motor Tic Disorder.

Subjects were scanned on a GE 1.5 Tesla Signa scanner on two consecutive weeks, before and after lithium. Prior to each scan all subjects received a YMRS (Young et al. 1978) and a modified Clinical Global Impression (CGI) for BPD (Spearing et al. 1997) completed by a child psychiatrist (PD), with parent's input. The parent(s) were allowed to remain in the scanner room with their children during the procedure. Although the manic subjects were motorically more hyperactive than the non-manic controls, with the exception of two subjects (requiring sedatives) there were no substantial differences among the preadolescents. They all presented with mild to moderate degree of anxiety inside the scanner, needing the assistance of their parents to entertain them during interval periods of the procedure.

The inpatient ward physician (blind to the study) titrated lithium according to a standard protocol. The loading dose was $30 \mathrm{mg} / \mathrm{kg}$, titrated by $300 \mathrm{mg}$ in children below $35 \mathrm{~kg}, 600 \mathrm{mg}$ in children between 25 and 40 $\mathrm{kg}$, and $900 \mathrm{mg}$ in patients above $40 \mathrm{~kg}$, every 3-5 days. The addition of lithium was assessed as a single variable, provided that all concomitant medications were maintained constant during the lithium titration. Patients with a CGI of 2 ("much improved") or above at week 1 were considered "responders" for purposes of a separate analysis. Two children who were severely manic received $25 \mathrm{mg}$ of oral thioridazine one hour before their scan.

Medication-free normal controls case-matched (one to one with patients) for age and gender were scanned and compared with their BPD counterparts at baseline. They received a medical and psychiatric history, mental status examination, as well as YMRS, CGI and a Children's Depression Rating Scale (CDRS) (Poznanski et al. 1979) to rule out any lifetime and present Axis I psychopathology.

\section{${ }^{1} \mathrm{H}$ MRS PROTOCOL}

All children and adolescents received a localized in vivo ${ }^{1} \mathrm{H}$ MRS scan while in a manic, a hypomanic, or a mixed episode. Landmark of the axial plane was done at the center of the forehead, $1-\mathrm{cm}$ above the eyebrows in all subjects to standardize heads positioning from scan to scan. To minimize head movements, the forehead was affixed with adhesive tape to the MRI stretcher and neck support was provided when necessary. Scans were repeated when motion artifacts were detected on the localizing scan, or when the linewidth clearly revealed a motion artifact. If necessary, scanning was interrupted and reinitiated after head adjustments were made and further support was offered to the patient. A short series of axial localizing images were acquired (Spin Echo, TR/TE $=500 \mathrm{~ms} / 14 \mathrm{~ms}, 3 \mathrm{~mm}$ contiguous slices, 256x192 matrix size, NEX $=0.75, \mathrm{FOV}=22 \mathrm{~cm}$, acquisition time $=1 \mathrm{~min}$ ) to maximize gray-white matter differentiation and measure specific regions of interest. A systematic approach to reference voxel positioning to identifiable anatomical landmarks in all subjects and controls was used. An axial cut approximately $1 \mathrm{~cm}$ above the genu of the corpus callosum showing a continuous view of the anterior and posterior horns of the lateral ventricles (Figure 1) was chosen as a reference slice. The center of a single $8 \mathrm{cc}$ voxel of predominantly gray (prefrontal) matter was centered on the frontal interhemispheric fissure, distal to the anterior horns of the lateral ventricles. Its proximal quadrant was placed 


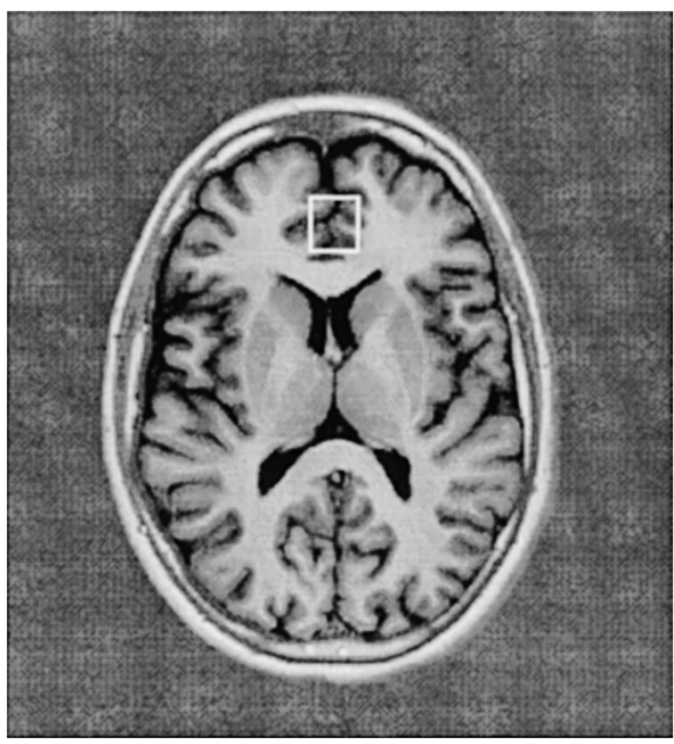

Figure 1. Voxel localization in anterior cingulate.

immediately adjacent to the zone of delimitation between white and gray matter (i.e. corpus callosum's margin with cingulate gyrus), in a prefrontal area corresponding to the rostral cingulate area, partially occupying rostral area 24 (Devinsky et al. 1995) and 32 of Brodman (Fuster 1999). This voxel placement was selected according to anatomical correlation with neuronal circuit or pathways postulated in the neurobiology of affective components of human behavior (Vogt 1993) and bipolar disorder (Pearlson 1999). Single voxel acquisitions were repeated if the quality of the spectra was not considered optimal. Any head movement was determined by observing the variation of the lineshape and amplitude of unsuppressed water (in magnitude mode) during manual pre-scan. Any head movement during data acquisition would be reflected from the linewidth of processed spectrum (in absorption mode). Sixty-four water-suppressed and four unsuppressed water signals were acquired $(\mathrm{TR} / \mathrm{TE}=3 \mathrm{~s} / 30 \mathrm{~ms}$, total acquisition time of $3.5 \mathrm{~min}$ ) using the PRESS sequence. When the full-width at half maximum (FWHM) of the water peak was greater than $15 \mathrm{~Hz}$, the water suppression was considered unsatisfactory, typically resulting in a poor baseline caused by the unsuppressed water tail. Such spectra were discarded, but a second ${ }^{1} \mathrm{H}$ MRS was acquired from the same location and included in the final analysis. This happened in approximately $50 \%$ of the cases. The unsuppressed water free-induction-decays (FIDs) were used to correct the phase of the metabolite spectra. Essentially, there was no operator bias introduced in phasing the mI peak with respect to NAA and choline and creatine: The phasing of the spectrum after the post water suppression was done automatically, using the tissue water FID, on a post processing macro de- veloped at UCLA, which includes Eddy current compensation, apodization, zero filling, Fourier transformation, and curve fitting and calculation of the ratios with respect to creatine. The reliability of measured data was controlled for by periodic calibration of the MR scanner against standard solutions of known concentration for spectroscopy.

\section{${ }^{1} \mathrm{H}$ MRS PROCESSING}

Data collection and analyses were conducted and interpreted in a blind manner by one research assistant (KY) trained in ${ }^{1} \mathrm{H}$ MRS acquisition and spectral analysis. Spectral processing of raw data done off-line on a Sun SPARC2 Workstation using SA/GE software (GE Systems, Milwaukee) consisted of a phase correction of the unsuppressed water FID, exponential apodization of $1.8 \mathrm{~Hz}$, zero-filling to 4096 complex points, and a Fast Fourier Transformation. Residual water signal was post-suppressed by a home-built algorithm. This software performs an automated (operator bias free) measurement of the areas under the curve of each of the spectral metabolite peaks for NAA, Cr, Cho and $\mathrm{mI}$. The area under each of the resonance peaks is assumed to be proportional to the brain concentration of each specific metabolite. They were selected automatically by a Threshold Averaging algorithm with a threshold setting at $1.90 \mathrm{E}+6$ units, and then Lorentzian-curve fitted using a Marquardt Algorithm with the peak table generated above as the initial guess. We assumed an exponential waveform for the apodized FID signals. After (fast) Fourier transformation, the spectral peaks would be presumably close to Lorentzian in shape, which were subsequently fitted. The specific bandwidth of water suppression in our sample was $75 \mathrm{~Hz}$ [FWHM]. We calculated the mean and standard deviations of the linewidths on the spectra of our subjects. The mean linewidth was $7.3 \mathrm{~Hz}$, and the standard deviation was 1.7 $\mathrm{Hz}$. As indicated above all linewidths above $15 \mathrm{~Hz}$ were discarded and reacquired. Results are reported in fitted areas under the curve over ratios of $\mathrm{Cr}$, a method well established in the field of ${ }^{1} \mathrm{H}$ MRS. In order to verify our assumption that $\mathrm{Cr}$ would not change with the experimental conditions we measured $\mathrm{Cr}$ to water ratios before and after lithium and found that the variation was only $1.8 \%$.

\section{DATA ANALYSIS}

Temporal changes (week 0 versus week 1 ) in metabolite resonances were assessed using Wilcoxon signedrank test considering non-normality of distribution of 
outcome scores. Specifically, the outcomes analyzed were the change between a baseline spectral measure and a repeat measure one week after lithium therapy on each metabolite within subjects, and the average metabolite resonances for subjects versus controls at baseline. We explored sensitivity of findings owing to the fact that matching between subjects and controls was based only on age and gender. First, findings involving controls that approached significance $(p<.10)$ were subjected to two-sample Mann-Whitney U-tests. Secondly, we evaluated the sensitivity of significant results to the possibility of hidden bias from unobserved covariates using the method of Rosenbaum (Rosenbaum 1991). With this method, an unobserved parameter " $\chi$ " is used to index the amount by which two units matched on the given characteristics (here, age and gender) might differ in their odds of being in the treated group. The goal is to identify the magnitude of " $\chi$ " that would be needed to overturn a significant result from a matched-pair analysis. Data analysis was done using SPSS.

\section{RESULTS}

Demographic aspects of the sample are displayed in Table 1. More boys than girls were scanned, and the age distribution showed a preponderance of preadolescents versus adolescents. Nine subjects were Caucasian and two were Caucasian-African-American. The mean serum lithium level at week 1 was $0.64 \mathrm{mEq} / \mathrm{L}$. Six patients showed a CGI score of 2 (much improved) at week 1 , and four patients showed a CGI score of 3 (minimally improved) at week 1 , after starting lithium therapy. Four of the six patients who had a CGI of 2 at week 1 had a lithium level $>0.5 \mathrm{mEq} / \mathrm{L}$. A 10 year-old patient with a serum level of $0.30 \mathrm{mEq} / \mathrm{L}$ showed a clinical response at week 1 , which was sustained and increased at week 3 after his lithium serum level, was increased to $0.5 \mathrm{mEq} / \mathrm{L}$.

Table 2 shows the results of the anterior cingulate cortex metabolites proton spectra reported in ratios of Cr. A significant $(p<.05)$ reduction in myo-inositol fitted area under the curve / $\mathrm{Cr}$ ratio was observed after 1 week of lithium therapy in children with BPD, as illustrated in Figure 2 and Figure 3. An increase in average myo-inositol/Cr ratio in subjects during the mixed manic phase compared to normal controls approached significance $(p=.054)$. Differences between subjects and controls were not significant for the other metabolites. A decrease in choline/ $\mathrm{Cr}$ after 1 week of lithium therapy did not reach statistical significance $(p<.131$ by Wilcoxon). Overall, NAA was the peak least affected by lithium. After removing a possible outlier the analysis still showed a strong trend of decreased myo-inositol $/ \mathrm{Cr}$ after lithium $(p<.075)$.

On sensitivity analysis (Rosenbaum 1991), we found that hidden bias corresponding to a $\chi$ value of less than 1.2 would still imply that the two-tailed $p$-value for comparison of treated and control values of myo-inositol would remain less than 0.1 , but for $\chi$ values of 1.25 or more, $p$-values less significant than 0.1 are plausible.

When responders and non-responders were compared at week 1 , defined by a CGI score of at least 2 (much improved), myo-inositol /Cr was decreased at one week versus baseline in the lithium-responder group $(p<.012$, Wilcoxon Signed Ranks Test), but not in the lithium-non-responder group $(p<.655$, Wilcoxon Signed Ranks Test).

A two-tailed Pearson's correlation coefficient between lithium blood levels and metabolite resonances before and after lithium therapy was attempted. Before calculating a correlation coefficient, the data was screened for outliers (which can cause misleading results) and evidence of a linear relationship was not found for any of the metabolites.

Table 1. Demographics

\begin{tabular}{|c|c|c|c|c|c|c|c|c|c|}
\hline Subject & Age & Gender & Diagnosis & Comorbidity & Medication(s) & $\begin{array}{c}\text { YMRS } \\
\text { Baseline/ } \\
1 \text { Week }\end{array}$ & $\begin{array}{c}\text { CGI } \\
\text { week } 1\end{array}$ & $\begin{array}{c}\text { Lithium } \\
\text { Level } \\
(\mathrm{mEq} / \mathrm{L})\end{array}$ & $\begin{array}{c}\text { CGI } \\
\text { week } 3\end{array}$ \\
\hline 1 & 7 & M & BP I, manic, rapid cycler & & Thioridazine & $26 / 18$ & 3 & 1.0 & 3 \\
\hline 2 & 8 & M & BP I, manic, rapid cycler & ADHD; Tics & Pimozide & $42 / 38$ & 3 & 0.6 & 2 \\
\hline 3 & 9 & M & BP I, manic & ADHD;ODD & Dextroamph; divalproex $\mathrm{Na}$ & $26 / 9$ & 2 & 0.6 & 2 \\
\hline 4 & 10 & M & BPII, hypomanic & ODD & & $14 / 12$ & 3 & 0.4 & 2 \\
\hline 5 & 10 & M & BP I, manic, rapid cycler & & Risperidone & $19 / 7$ & 2 & 0.3 & 2 \\
\hline 6 & 10 & M & BP I, manic, rapid cycler & ADHD; Diabetes & Dextroamph; insulin & $25 / 16$ & 2 & 1.0 & 2 \\
\hline 7 & 11 & $\mathrm{~F}$ & BP I, manic, rapid cycler & & Risperidone & $16 / 8$ & 2 & 0.7 & 2 \\
\hline 8 & 13 & M & BP II, hypomanic & ADHD;ODD & Dextroamph; carbamazep. & $25 / 17$ & 2 & 0.5 & 2 \\
\hline 9 & 15 & M & BPI, manic & ADHD;ODD & Dextroamph & $29 / 17$ & 2 & 0.6 & 3 \\
\hline 10 & 16 & M & BP I, manic, rapid cycler & & Olanzapine & $15 / 15$ & 3 & 0.8 & 3 \\
\hline 11 & 17 & $\mathrm{~F}$ & BP I, manic & & & $23 / 5$ & 1 & 0.6 & 1 \\
\hline
\end{tabular}


Table 2. Mean Pre-treatment ${ }^{1} \mathrm{H}$ MRS Metabolites/Creatine vs. Post-treatment ${ }^{1} \mathrm{H}$ MRS Metabolites/Creatine in Children and Adolescents with Bipolar Disorder before and after Lithium, and Normal Controls.

\begin{tabular}{lclcr}
\hline Frontal (AUC) & mI/Cr & Cho/Cr & Glx/Cr & NAA/Cr \\
\hline Without LiC03 & $1.092 \pm .612$ & $.917 \pm .261$ & $4.157 \pm 1.565$ & $1.373 \pm .381$ \\
With LiC03 & $.820 \pm .279$ & $.789 \pm .111$ & $3.829 \pm .742$ & $1.262 \pm .123$ \\
$p$-value (Wilcoxon) & $0.047^{*}$ & .131 & .594 & .657 \\
Controls & $.821 \pm .152$ & $.800 \pm .171$ & $4.311 \pm 1.402$ & $1.233 \pm .189$ \\
$p$-value (Wilcoxon) & .075 & .424 & .790 & .477 \\
\hline
\end{tabular}

\section{DISCUSSION}

Results from our study show evidence of a significant decrease in anterior cingulate myo-inositol / Cr ratios following seven days of lithium therapy in children and adolescents with early-onset BPD. The observed association and possible reduction in anterior cingulate cortex myo-inositol with lithium is consistent with in vitro studies of lithium's action (Manji et al. 1996). It is also consistent with a recent report of decreased myo-inositol levels after 5-7 days of acute lithium treatment, and at week 4, in sample of adults with BPD (Moore et al. 1999), suggesting that in vivo changes in anterior cingulate myo-inositol resonance after lithium in patients with BPD demonstrate consistency across development. The relevance of myo-inositol changes measured

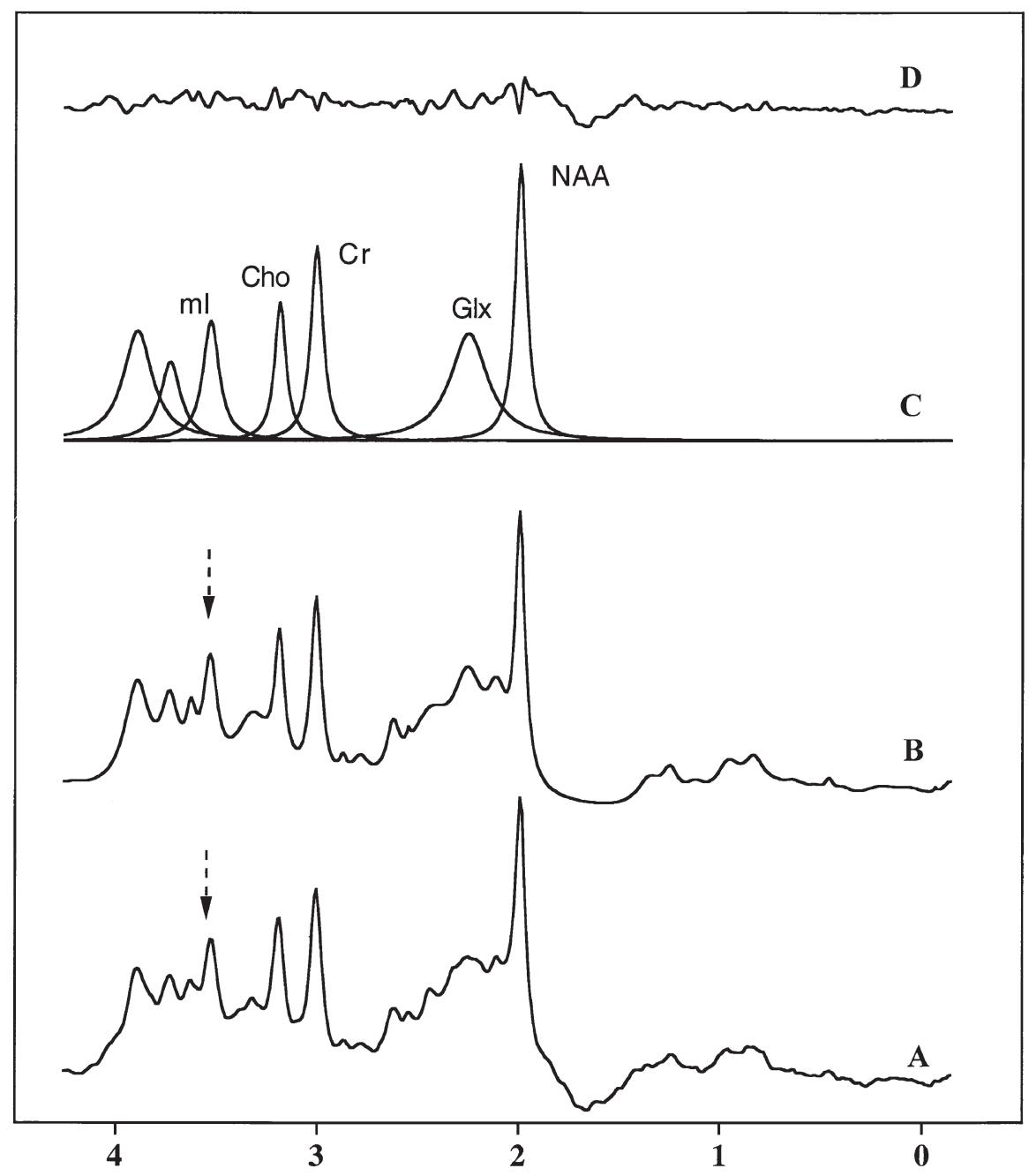

Chemical Shift, ppm
Figure 2. ${ }^{1} \mathrm{H}$ MRS spectra of cingulate area in a manic pre-adolescent with BPD after lithium therapy: A-raw spectrum; B-fitted spectrum; C-simulated deconvoluted spectra; D-difference between A-B. 


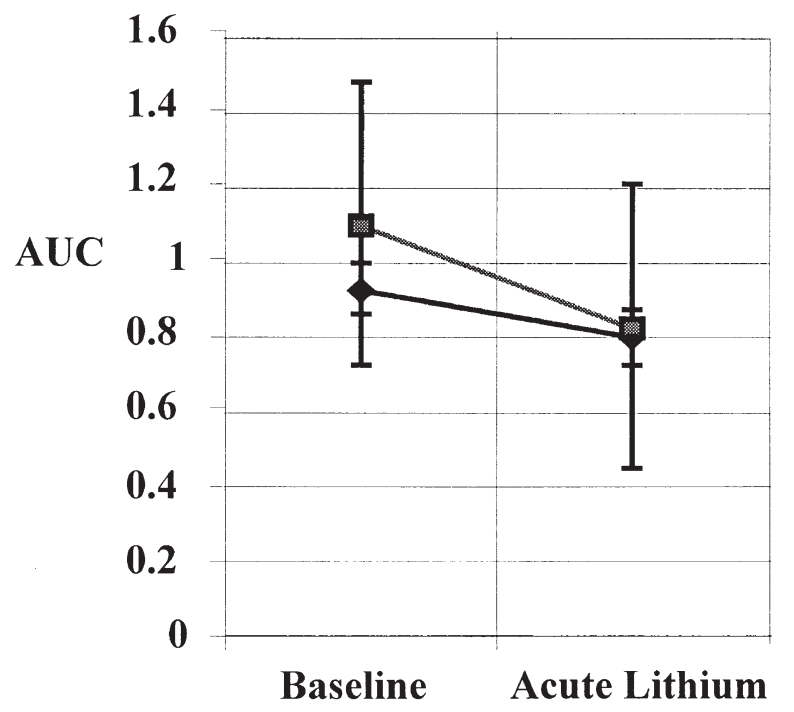

Cho: $\mathrm{p}<0.131$

Acute Lithium Vs Baseline

(Wilcoxon)

\section{$\smile$ Choline}

$\rightarrow$ Myo-inositol

mI: $\mathbf{p}<0.047$

Acute Lithium Vs Baseline

(Wilcoxon)

Figure 3. Cingulate mean ${ }^{1} \mathrm{H} \mathrm{MRS} \mathrm{mI} / \mathrm{Cr}$ and $\mathrm{Cho} / \mathrm{Cr}$ in children with $\mathrm{BPD}$ at baseline and after acute lithium treatment.

in this location is supported by clinical syndromes associated with identifiable anatomic locations (Strakowski et al. 1999) manifesting with emotional dysregulation, and by studies (George et al. 1998; Ketter et al. 1996; Mayberg et al. 1999) involving healthy volunteers showing brain activation induced by mood states. Disruptions in this limbic-thalamic-prefrontal cortical circuit (Cummings 1995) may contribute to the behavioral syndromes associated with BPD.

A comparison of effect sizes estimated from the Moore adult study (Moore et al. 1999) and our data shows that for myo-inositol / Cr, our measured effect size (taking the observed mean difference in area under the curve divided by the standard deviation of the differences), was 1.0, yielding a result that just achieved statistical significance. In contrast, Moore's findings (Moore et al. 1999) implied a larger effect size of 2.6. Their study reflects myo-inositol levels and not myoinositol /Cr ratio, which may explain the differences in effect size, nonetheless still showing a robust effect. It is possible that non-significant findings on other variables in our study could be explained by a lack of power in the available sample, as the required sample size for $80 \%$ power with alpha $=0.05$ would be 15 for an effect size of $0.8,24$ for an effect size of 0.6 , and 52 for an effect size of 0.4 .

Our results differ from those of Silverstone and collaborators, who did not report any significant effects of seven days of lithium administration on myo-inositol levels in the temporal lobes of healthy volunteers (Silverstone et al. 1996). This study has a number of methodological differences compared to ours, mainly the inclusion of healthy volunteers rather than patients with $\mathrm{BPD}$, and the choice of temporal lobes versus anterior cingulate as a region of interest.
It is possible that the pre-to-post lithium reduction in myo-inositol / $\mathrm{Cr}$ in our sample was skewed to some degree by the very high myo-inositol / Cr ratio at baseline in one subject. After removing this subject's data from the analysis the decrease in myo-inositol / Cr post lithium was no longer statistically significant but a strong decrease of the myo-inositol / Cr ratio after lithium remained. This pattern of myo-inositol $/ \mathrm{Cr}$ decrease post-lithium was observed in eight of the ten remaining subjects.

That six patients had comorbid diagnoses, the most common being ADHD, is typical of the psychopathology of early-onset BPD (Biederman et al. 1996). The presence of a non-affective childhood psychiatric diagnosis does not diminish the likelihood of a therapeutic response to lithium among adolescents with acute mania, although the potential trait-effect ADHD may have as a condition on the proton spectra of five patients with comorbid ADHD constitutes a limitation of this study.

Uncertainty over developmental modifications of diagnostic criteria for early onset BPD may lessen the conclusions pertaining to biological measures in this population. A lack of clear definitions regarding the severity and characteristics of classic mania as it applies to children has contributed to the blurring of diagnostic boundaries in early onset BPD. Nevertheless, the somewhat atypical clinical presentation of juvenile-onset $\mathrm{BPD}$, including extreme hyperactivity, moodiness, chronic rather than episodic irritability and prolonged temper tantrums (Geller and Luby 1997), alternating with depressive symptoms and difficulty sleeping at night (Weller et al. 1995), differentiates children diagnosed with BPD in our sample from children with ADHD (Biederman et al. 1996). Although some of these 
symptoms-hyperactivity, distractibility, impulsive risk taking-prevail in both BPD and ADHD, testing with standard screening rating scales also show that delinquent and aggressive behavior, somatic complaints, anxiety/depression, and thought problems are far more severe in children with BPD than ADHD (Biederman et al. 1995).

Significant differences between subjects and controls were not found for any of the metabolites. A near significant difference was seen for myo-inositol in subjects during the manic phase compared to normal controls. The lack of significance of differences between patients and controls may be due to lack of statistical power due to the small size of this sample. From our sensitivity analysis we conclude that, because it is plausible that an unobserved characteristic that is not controlled in the matching might confer an odds ratio of 1.25 in favor of being in the treated rather than the control arm, given that an odds ratio of 1.25 is not very large a priori, there is a considerable amount of sensitivity in the significance of the results we report. This is not surprising, however, based as they are on a very modest sample size.

The potential identification of a biological marker of treatment response continues to have importance for both children and adults with BPD. One biological (Stoll et al. 1991) and several clinical (Bowden 1995; Calabrese et al. 1996) predictors of acute lithium treatment response have been reported in adults. Severity of manic versus mixed (Calabrese et al. 1996), and depressive (Swann et al. 1997) symptomatology, prior response to lithium, and infrequent episodes (Bowden 1995) have been associated with lithium responsiveness in adults with BPD. Similarly, elevated erythrocyte choline levels has been correlated with less likelihood of response to lithium monotherapy in a subgroup of perhaps more severely ill patients (Stoll et al. 1991). Confounders such as definition of response, mood state, prior lithium exposure, and compliance have undermined the literature with inconsistencies. Examining our lithium responders, in patients with a CGI of 2 ("much improved") or above at week 1 (scanned during a manic phase, none with prior lithium exposure), a pattern of early decrease in myo-inositol / Cr after one week of lithium may portend a good therapeutic response. Accordingly, Moore and colleagues (Moore et al. 1999), have suggested that an acute decrement in the prefrontal myo-inositol in vivo resonance may indicate a successful alteration of the cascade of secondary messenger signaling and possible gene expression changes ultimately associated with clinical efficacy. In this sense, BPD children who display reduction in anterior cingulate myo-inositol signal early on after initiation of lithium therapy may be predicted to show a better treatment response versus those in which myo-inositol shows no significant change after acute lithium therapy. This hypothesis needs to be demonstrated with a larger sample, especially since changes in myo-inositol might be related to improvement in mania and not to lithium administration per se. In two of our responder subjects (males, ages 10 and 8; lithium levels $0.4 \mathrm{mEq} / \mathrm{L}$ and 0.6 $\mathrm{mEq} / \mathrm{L}$ ) myo-inositol signal changes following lithium administration occurred before clinical changes in mania ratings, suggesting the detection of a biochemical signal prior to clinical change. Nevertheless, in the remaining five responders (mean lithium $0.7 \mathrm{mEq} / \mathrm{L}$ ) improvement in mania ratings were measured at week 1.

In summary, this preliminary report lends support to existing evidence of lithium's suppressive effect on the inositol system. Although no significant differences were seen in metabolite ratios between subjects and controls, there was a strong trend towards increased myo-inositol / $\mathrm{Cr}$ and choline/ $\mathrm{Cr}$ in lithium naive subjects versus controls.

Limitations of the study include small sample size, use of a single voxel for anatomical location, and only one repeat MRS assessment following start of lithium therapy. Scanning a second region in the brain, one not thought to be involved in the neurophysiology of BPD, could have shown if myo-inositol changes are regionally specific, and yet the clinical status of the subjects did not allow for a prolonged MRS acquisition.

No adjustment has been made to the results for multiple comparisons. Given the available sample size, it is not possible to rule out that the significant findings we observed were actually Type I errors, i.e., the most significant results across several comparisons with no underlying differences between groups. Nonetheless, given the exploratory nature of the study, we do not regard adjustment for multiple comparisons as desirable, as such adjustments can give rise to Type II errors (erroneous findings of non-significance).

It has been reported that at $1.5 \mathrm{~T}$ field strength, the MRS peak due to glycine overlaps with the myo-inositol peak at 3.54ppm (Miller 1991). We acknowledge, therefore, the potential confounder presented by the resonances of glycine and inositol-1-phophate on the myo-inositol resonance peak. Nevertheless, concordant with Moore and colleagues (Moore et al. 1999) and on the basis of prior findings (Ross et al. 1994) we believe these compounds contribute a minor $(<5 \%)$ component to the myo-inositol resonance. The proximity of $\mathrm{mI}$ to the water resonance may also constitute a confounder. Nevertheless, water resonates at $4.8 \mathrm{ppm}$ and $\mathrm{mI}$ resonates at $3.5 \mathrm{ppm}$, therefore they are $83 \mathrm{~Hz}$ away from each other in the normal spectrum. The specific bandwidth of water suppression in our sample was 75 $\mathrm{Hz}$, conceivably not affecting the myo-inositol desaturate or suppressing the myo-inositol peak significantly. The current data is analyzed under the assumption of a complete Lorentzian line shape for $\mathrm{mI}$. In our future measures a combined Lorentzian /gaussian line shape for fitting the peaks will be utilized. 
An additional limitation of this study is the paucity of data concerning a possible developmental variability in metabolite resonances across childhood and adolescence. Although not observed in our small sample, age-related changes have been described for myo-inositol and for choline in very young children, with myo-inositol found to be very prominent in spectra at birth, and choline increasingly seen in older infants (Kreis et al. 1993). Studies are underway to clarify the developmental influences on proton spectra resonances in the anterior cingulate cortex, given the structural changes observed during development (Giedd et al. 1996) in this and other related areas of the brain correlated with mood regulation. Also, more information on the possible influence of psychotropics on metabolite peaks is needed. Since we do not know if spectroscopic findings are state or trait-related in this population, the potential independent effect of a diagnosis of ADHD needs to be explored. Future studies are clearly needed to further investigate the relationships between clinical characteristics, baseline brain metabolism spectra, structural variation, and treatment response.

\section{ACKNOWLEDGMENTS}

This project was funded by a National Alliance for Research on Schizophrenia and Depression (NARSAD) Young Investigator Award, 1998-99; and a National Institute of Mental Health (NIMH); K01 MH01601-01, Career Developmental Award to PD, and by a Stanley Foundation grant to JTM. We are grateful to Peter Whybrow, MD, Stephen Strakowski, MD, Perry Renshaw, MD, Gregory Moore, PhD, Jillian Kleiner, MD, Robert Kowatch, MD, and Xavier Castellanos, MD for their valuable comments about the project. We also thank Bhavik Shah, MD; Mark de Antonio, MD; and Dushon Blackburn-Ryan for their support with patient recruitment. Presented in part at the 37th Annual Meeting of the American College of Neuropsychopharmacology, Las Croabas, Puerto Rico, December 14-18, 1998.

\section{REFERENCES}

Atack JR, Broughton HB, Pollack SJ (1995): Inositol monophosphatase - a putative target for $\mathrm{Li}+$ in the treatment of bipolar disorder. Trends Neurosci 18:343-349

Berridge MJ, Downes CP, Hanley MR (1982): Lithium amplifies agonist-dependent phosphatidylinositol responses in brain and salivary glands. Biochem J 206:587-595

Biederman J, Faraone S, Mick E, Wozniak J, Chen L, Ouellette C, Marrs A, Moore P, Garcia J, Mennin D, Lelon E (1996): Attention-deficit hyperactivity disorder and juvenile mania: an overlooked comorbidity? J Am Acad Child Adolesc Psychiatry 35:997-1008

Biederman J, Wozniak J, Kiely K, Ablon S, Faraone S, Mick E, Mundy E, Kraus I (1995): CBCL clinical scales discriminate prepubertal children with structured interviewderived diagnosis of mania from those with ADHD. J Am Acad Child Adolesc Psychiatry 34:464-471
Bowden CL (1995): Predictors of response to divalproex and lithium. J Clin Psychiatry 56 Suppl 3:25-30

Brown AS, Mallinger AG, Renbaum LC (1993): Elevated platelet membrane phosphatidylinositol-4,5-bisphosphate in bipolar mania. Am J Psychiatry 150:1252-1254

Calabrese JR, Fatemi SH, Kujawa M, Woyshville MJ (1996): Predictors of response to mood stabilizers. J Clin Psychopharmacol 16:24S-31S

Carlson GA, Rapport MD, Pataki CS, Kelly KL (1992): Lithium in hospitalized children at 4 and 8 weeks: mood, behavior and cognitive effects. J Child Psychol Psychiatry 33:411-425

Costello EJ, Angold A, Burns BJ, Stangl DK, Tweed DL, Erkanli A, Worthman CM (1996): The Great Smoky Mountains Study of Youth. Goals, design, methods, and the prevalence of DSM-III-R disorders. Arch Gen Psychiatry 53:1129-1136

Cummings JL (1995): Anatomic and behavioral aspects of frontal-subcortical circuits. Ann N Y Acad Sci 769:1-13

del Rio E, Shinomura T, van der Kaay J, Nicholls DG, Downes CP (1998): Disruption by lithium of phosphoinositide signalling in cerebellar granule cells in primary culture. J Neurochem 70:1662-1669

Devinsky O, Morrell MJ, Vogt BA (1995): Contributions of anterior cingulate cortex to behaviour. Brain 118:279-306

Fava M (1997): Psychopharmacologic treatment of pathologic aggression. Psychiatr Clin North Am 20:427-451

Feldman RS, Meyer JS, Quenzer LF (1997): Principles of neuropsychopharmacology. Sunderland, MA, Sinauer Associates

Friedman E, Hoau Yan W, Levinson D, Connell TA, Singh H (1993): Altered platelet protein kinase C activity in bipolar affective disorder, manic episode. Biol Psychiatry 33:520-525

Fuster JM (1999): Synopsis of function and dysfunction of the frontal lobe. Acta Psychiatr Scand Suppl 395:51-57

Geller B, Cooper TB, Sun K, Zimerman B, Frazier J, Williams M, Heath J (1998): Double-blind and placebo-controlled study of lithium for adolescent bipolar disorders with secondary substance dependency. Journal of the American Academy of Child and Adolescent Psychiatry 37:171-178

Geller B, Luby J (1997): Child and adolescent bipolar disorder: a review of the past 10 years. Journal of the American Academy of Child and Adolescent Psychiatry 36:1168-1176

George MS, Huggins T, McDermut W, Parekh PI, Rubinow D, Post RM (1998): Abnormal facial emotion recognition in depression: serial testing in an ultra-rapid-cycling patient. Behav Modif 22:192-204

Giedd JN, Vaituzis AC, Hamburger SD, Lange N, Rajapakse JC, Kaysen D, Vauss YC, Rapoport JL (1996): Quantitative MRI of the temporal lobe, amygdala, and hippocampus in normal human development: ages 4-18 years. J Comp Neurol 366:223-230

Gonzalez RG, Guimaraes AR, Sachs GS, Rosenbaum JF, Garwood M, Renshaw PF (1993): Measurement of human brain lithium in vivo by MR spectroscopy. AJNR Am J Neuroradiol 14:1027-1037

Himmelhoch JM, Garfinkel ME (1986): Sources of lithium 
resistance in mixed mania. Psychopharmacol Bull 22:613-620

Hsu LK (1986): Lithium-resistant adolescent mania. J Am Acad Child Psychiatry 25:280-283

Kato T, Hamakawa H, Shioiri T, Murashita J, Takahashi Y, Takahashi S, Inubushi T (1996): Choline-containing compounds detected by proton magnetic resonance spectroscopy in the basal ganglia in bipolar disorder. J Psychiatry Neurosci 21:248-254

Kato T, Shioiri T, Inubushi T, Takahashi S (1993): Brain lithium concentrations measured with lithium-7 magnetic resonance spectroscopy in patients with affective disorders: relationship to erythrocyte and serum concentrations. Biol Psychiatry 33:147-152

Kato T, Shioiri T, Murashita J, Hamakawa H, Takahashi Y, Inubushi T, Takahashi S (1995): Lateralized abnormality of high energy phosphate metabolism in the frontal lobes of patients with bipolar disorder detected by phase-encoded 31P-MRS. Psychol Med 25:557-566

Ketter TA, Andreason PJ, George MS, Lee C, Gill DS, Parekh PI, Willis MW, Herscovitch P, Post RM (1996): Anterior paralimbic mediation of procaine-induced emotional and psychosensory experiences. Arch Gen Psychiatry 53:59-69

Kreis R, Ernst T, Ross BD (1993): Development of the human brain: in vivo quantification of metabolite and water content with proton magnetic resonance spectroscopy. Magn Reson Med 30:424-437

Lewinsohn PM, Klein DN, Seeley JR (1995): Bipolar disorders in a community sample of older adolescents: prevalence, phenomenology, comorbidity, and course. J Am Acad Child Adolesc Psychiatry 34:454-463

Manji HK, Bersudsky Y, Chen G, Belmaker RH, Potter WZ (1996): Modulation of protein kinase $C$ isozymes and substrates by lithium: the role of myo-inositol. Neuropsychopharmacology 15:370-381

Mayberg HS, Liotti M, Brannan SK, McGinnis S, Mahurin RK, Jerabek PA, Silva JA, Tekell JL, Martin CC, Lancaster JL, Fox PT (1999): Reciprocal limbic-cortical function and negative mood: converging PET findings in depression and normal sadness. Am J Psychiatry 156:675-682

Miller BL (1991): A review of chemical issues in 1H NMR spectroscopy: N-acetyl-L-aspartate, creatine and choline. NMR Biomed 4:47-52

Moore GJ, Bebchuk JM, Parrish JK, Faulk MW, Arfken CL, Strahl-Bevacqua J, Manji HK (1999): Temporal dissociation between lithium-induced changes in frontal lobe myo-inositol and clinical response in manic-depressive illness. Am J Psychiatry 156:1902-1908

Murray M, Greenberg ML (1997): Regulation of inositol monophosphatase in Saccharomyces cerevisiae. Mol Microbiol 25:541-546

Pearlson GD (1999): Structural and functional brain changes in bipolar disorder: a selective review. Schizophr Res 39:133-140

Poznanski EO, Cook SC, Carroll BJ (1979): A depression rating scale for children. Pediatrics 64:442-450

Renshaw PF, Yurgelun-Todd DA, Tohen M, Gruber S, Cohen BM (1995): Temporal lobe proton magnetic resonance spectroscopy of patients with first-episode psychosis. Am J Psychiatry 152:444-446
Rosenbaum, P.R. (1991): Discussing hidden bias in observational studies. Annals Int Medicine 115: 901-905

Ross BD, Jacobson S, Villamil F, Korula J, Kreis R, Ernst T, Shonk T, Moats RA (1994): Subclinical hepatic encephalopathy: proton MR spectroscopic abnormalities. Radiology 193:457-463

Ryan ND, Bhatara VS, Perel JM (1999): Mood stabilizers in children and adolescents. J Am Acad Child Adolesc Psychiatry 38:529-536

Sachs GS, Renshaw PF, Lafer B, Stoll AL, Guimaraes AR, Rosenbaum JF, Gonzalez RG (1995): Variability of brain lithium levels during maintenance treatment: a magnetic resonance spectroscopy study. Biol Psychiatry $38: 422-428$

Sharma R, Venkatasubramanian PN, Barany M, Davis JM (1992): Proton magnetic resonance spectroscopy of the brain in schizophrenic and affective patients. Schizophr Res 8:43-49

Sheehan DV, Lecrubier Y, Sheehan KH, Amorim P, Janavs J, Weiller E, Hergueta T, Baker R, Dunbar GC (1998): The Mini-International Neuropsychiatric Interview (M.I.N.I.): the development and validation of a structured diagnostic psychiatric interview for DSM-IV and ICD-10. J Clin Psychiatry 59:22-33; quiz 34-57

Silverstone PH, Hanstock CC, Fabian J, Staab R, Allen PS (1996): Chronic lithium does not alter human myo-inositol or phosphomonoester concentrations as measured by $1 \mathrm{H}$ and 31P MRS. Biol Psychiatry 40:235-246

Soares JC, Mallinger AG, Dippold CS, Frank E, Kupfer DJ (1999): Platelet membrane phospholipids in euthymic bipolar disorder patients: are they affected by lithium treatment? Biol Psychiatry 45:453-457

Spearing MK, Post RM, Leverich GS, Brandt D, Nolen W (1997): Modification of the Clinical Global Impressions (CGI) Scale for use in bipolar illness (BP): the CGI-BP. Psychiatry Res 73:159-171

Stoll AL, Cohen BM, Snyder MB, Hanin I (1991): Erythrocyte choline concentration in bipolar disorder: a predictor of clinical course and medication response. Biol Psychiatry 29:1171-1180

Strakowski SM, DelBello MP, Sax KW, Zimmerman ME, Shear PK, Hawkins JM, Larson ER (1999): Brain magnetic resonance imaging of structural abnormalities in bipolar disorder. Arch Gen Psychiatry 56:254-260

Strober M, Schmidt-Lackner S, Freeman R, Bower S, Lampert C, DeAntonio M (1995): Recovery and relapse in adolescents with bipolar affective illness: a five-year naturalistic, prospective follow-up. J Am Acad Child Adolesc Psychiatry 34:724-731

Swann AC, Bowden CL, Morris D, Calabrese JR, Petty F, Small J, Dilsaver SC, Davis JM (1997): Depression during mania. Treatment response to lithium or divalproex. Arch Gen Psychiatry 54:37-42

Tondo L, Baldessarini RJ, Hennen J, Floris G, Silvetti F, Tohen M (1998): Lithium treatment and risk of suicidal behavior in bipolar disorder patients. J Clin Psychiatry 59:405-414

Vitiello B, Stoff DM (1997): Subtypes of aggression and their relevance to child psychiatry. J Am Acad Child Adolesc Psychiatry 36:307-315 
Vogt BA (1993): Structural organization of cingulate cortex: areas, neurons, and somatodendritic receptors. In: Vogt BA, Gabriel M, (eds) Neurobiology of Cingulate Cortex and Limbic Thalamus: a Comprehensive Handbook. Boston, Birkhauser, pp. 19-70

Weller EB, Weller RA, Fristad MA (1995): Bipolar disorder in children: misdiagnosis, underdiagnosis, and future directions. J Am Acad Child Adolesc Psychiatry 34:709_ 714

Young RC, Biggs JT, Ziegler VE, Meyer DA (1978): A rating scale for mania: reliability, validity and sensitivity. Br J Psychiatry 133:429-435 